\title{
MORFOLOGÍA E HISTOLOGÍA DEL APARATO REPRODUCTOR DE SUpputius cincticeps (HEMIPTERA: HETEROPTERA: PENTATOMIDAE)
}

\section{MORPHOLOGY AND HISTOLOGY OF THE REPRODUCTIVE SYSTEM OF Supputius cincticeps (HEMIPTERA: HETEROPTERA: PENTATOMIDAE)}

\author{
Alberto Soto ${ }^{1}$, Hamilton G. Oliveira², Tito Bacca ${ }^{3}$
}

${ }^{1}$ I.A., M.Sc., Ph.D. Profesor Departamento de Producción Agropecuaria. Universidad de Caldas. Calle 65 No 26-10 Manizales, Caldas, Colombia. E-mail: alberto.soto@ucaldas.edu.co 2 Ph.D. Investigador Centro Nacional de Investigaciones en Palma, CENIPALMA, Villavicencio, Colombia. E-mail: hgomes@cenipalma.org 3 I.A., M.Sc., Ph.D. Profesor Asociado Universidad de Nariño, Facultad de Ciencias Agrícolas, Torobajo, Pasto, Nariño, Colombia. E-mail: titobacca@gmail.com

Rev. U.D.C.A Act \& Div. Cient. 15(1): 117 - 123, 2012

\section{RESUMEN}

Los insectos plaga representan los mayores problemas en plantaciones forestales, principalmente, en monocultivos del género Eucalyptus, debido a los relevantes daños y su persistencia durante todo el ciclo de cultivo. Supputius cincticeps presenta potencial para ser usado en el control biológico de larvas defoliadoras de eucalipto. El objetivo de este trabajo fue estudiar la morfología e histología de los órganos reproductivos de S. cincticeps, con el fin de contribuir en el conocimiento de su reproducción, para ser aplicado en programas de cría masiva. Los ovarios y los testículos de ese depredador fueron extraídos y se realizaron cortes de $5 \mathrm{~mm}$, en un micrótomo automático, los cuales, fueron descritos y registrados fotográficamente.

Palabras clave: Depredador, genitalia, macho, hembra, chinche.

\section{SUMMARY}

Insect pests represent the most remarkable problems in forest plantations. This scenario is present mainly in monocultures of the genus Eucalyptus, due to significant damage that occurs throughout the growing season. Supputius cincticeps might be used biological control agent of eucalyptus defoliating larvae. The aim of this work was to study the morphology and histology of the reproductive organs of S. cincticeps to contribute to the knowledge of their reproduction to be applied in mass rearing programs. Ovaries and testicles of the predator were extracted and sectioned by using a microtome up to obtain tissue sections of $5 \mathrm{~mm}$ which were described and recorded photographically.

Key words: Predator, genitalia, male, female, stink bug.

\section{INTRODUCCIÓN}

El incremento exagerado de poblaciones de lepidópteros defoliadores causa perjuicios en plantaciones de eucalipto en Brasil, en especial, la especie Thyrinteina arnobia (Stoll) (Lepidoptera: Geometridae) (Santos et al. 1993; Zanuncio et al. 2005a; Pereira et al. 2008). Debido a que el control químico de esos insectos aumenta los costos de producción y causa impacto ambiental, se han estudiado otras alternativas de control; una de ellas es el control biológico de plagas (Zanuncio et al. 2005b). La utilización de parasitoides y de depredadores en programas de control biológico aplicado, requiere la obtención, mediante crías masales de estos agentes (De Clercq et al. 2002). Para esto, se ha incrementado el uso de chinches predadores de la familia Pentatomidae (Hemiptera), por ser importantes enemigos naturales generalistas de plagas agrícolas, ser voraces, con alta capacidad reproductiva y alta tasa de depredación, principalmente, de larvas de Lepidoptera y Coleoptera (Oliveira et al. 2002; Zanuncio et al. 2005b; Holtz et al. 2006). Los géneros Brontocoris y Podisus (Hemiptera: Heteroptera: Pentatomidae) son producidos y liberados en programas de control biológico de lepidópteros defoliadores de eucalipto 
(Zanuncio et al. 2002). Supputius cincticeps Stal, 1860 es predador del defoliador del eucalipto Sabulodes caberata Guenée, 1857 (Lepidoptera: Geometridae) y sus ninfas también predan larvas de Alabama arguillacea (Lepidoptera: Noctuidae) (Zanuncio et al. 2002; Holtz et al. 2006). Esta especie predadora ha sido encontrada en baja densidad en reforestaciones con eucalipto, lo que hace necesario el desarrollo de estudios sobre su biología, reproducción y cría masiva, para que este predador pueda ser multiplicado y liberado en programas de control biológico (Zanuncio et al. 1996-2000). Estudios realizados por Zanuncio et al. 2005a, concluyen que $S$. cincticeps presenta alta factibilidad para ser usado en el control biológico de larvas defoliadoras de eucalipto y que su producción masiva en insectarios puede ser desarollada, con facilidad, utilizándo como presas, larvas de la mosca doméstica Musca domestica (Diptera: Muscidae) o de Tenebrio molitor (Coleoptera: Tenebrionidae).

Algunas empresas forestales se han dedicado a la producción masiva de estos predadores, con el objetivo de incrementar artificialmente su densidad poblacional (Zanuncio et al. 1992).

De esta manera, los conocimientos sobre la biología y la potencialidad reproductiva de estos predadores son de suma importancia, con el fin de desarrollar metodologías eficientes de multiplicación de esos insectos.

El aparato reproductor masculino de la mayoría de los insectos presenta glándulas accesorias, que se abren en el canal deferente o en el ducto eyaculatorio (Happ, 1992; Chapman, 1998). En general, cada testículo está compuesto por un número de tubos o folículos testiculares, variando de uno en algunos Coleoptera a más de cien en grillos (Acrididae) (Nijhout, 1994; Chapman, 1998). Cada folículo tiene su propio germario, análogo a los ovaríolos femeninos, excepto por el revestimiento común de los folículos, con aspecto de cuerpo compacto (Nijhout, 1994). El aparato reproductor de machos de Heteroptera consiste, típicamente, de un par de gónadas conectadas con vesículas seminales pareadas y un ducto eyaculatorio mediano (Nijhout, 1994; Chapman, 1998).

El aparato reproductor femenino de insectos consiste en un par de ovarios ubicado en la parte superior o lateral del sistema digestivo, formados por unidades funcionales, denominadas ovaríolos, ligados por el pedicelo a los oviductos laterales y éstos al oviducto común, que se abre en una cámara genital llamada vagina y de ahí para el exterior, en la bursa copulatrix (Nijhout, 1994; Chapman, 1998).

Cada ovario está formado por la región del germario en la parte distal, con células en proceso inicial de diferenciación. El trofario o cámara trófica es un conjunto de células nutrientes que envuelven la parte central del ovaríolo, llamado el cordón nutritivo y que componen el sistema de transporte de nutrien- tes entre los trofocitos y los ovocitos. Otra región del ovario está constituida por un vitelario, donde ocurre el desarrollo de la vitelogénesis y de los ovocitos en disposición linear, con diferentes estados de desarrollo (Nijhout, 1994; Chapman, 1998). Cada ovario, típicamente, contiene cuatro a siete ovaríolos, mas este número es especie-específico y, a veces, familia-específico, pudiendo ser de uno, como en algunos coleópteros, hasta dos mil, en reinas de termitas Eutermes sp. (Isoptera: Termitidae) (Buning, 1994; Nijhout, 1994; Chapman, 1998). Esta variación ocurre en función del modo de vida, relaciones filogenéticas y tamaño del insecto (Chapman, 1998).

La estructura de los ovaríolos de los Heteroptera es uniforme, con diferencias en la organización del trofario (cámara trófica). Estudios morfológicos, estructurales e histoquímicos muestran diferencias en los trofarios de Heteroptera de especies con diferente posición filogenética (Ksiazkiewicz-Kapraskla, 1985; 1991; Simiczyjew et al. 1998; Szklarzewicz, 1998). La morfología del sistema reproductor femenino de Podisus nigrispinus Dallas, 1851 (Heteroptera: Pentatomidae) presenta gónadas internas de coloración amarillenta y, en cada ovario, se observan siete ovaríolos unidos por los filamentos terminales, en una estructura en forma de cuerno (Lemos et al. 2005). El conocimiento de la morfología reproductiva de los enemigos naturales y principalmente de las hembras es fundamental en su cría masiva, debido a que es posible conocer la dinámica de producción de huevos, factor crucial en la producción de entomófagos. El estado de desarrollo de los ovarios puede determinar la duración del periodo de preoviposición, tasa de oviposición, frecuencia y duración de alimentación antes de la oviposición y repuesta de estímulos externos, para la producción y maduración de huevos (Jervis et al. 2005). Por lo tanto, conocer el estado de desarrollo los huevos puede ser un parámetro importante, cuando se realicen crías masivas del depredador S. cincticeps.

El objetivo de este trabajo fue describir anatómica y morfológicamente algunas estructuras del aparato reproductor de machos y de hembras de S. cincticeps.

\section{MATERIALES Y MÉTODOS}

El estudio, se realizó en el laboratorio de Biología Molecular y Celular del Departamento de Biología General, de la Universidade Federal de Viçosa, Minas Gerais, Brazil. Los adultos sexualmente maduros de $S$. cincticeps fueron obtenidos de la cría masal del insectario del Departamento de Biología Animal, sexados por la apariencia externa de la genitalia y por el tamaño del cuerpo, siendo la hembra de mayor tamaño y más ancha a nivel del pronoto, con respecto al macho. 
Los adultos, se colocaron en frascos con fijador Zambonini Stefanini, pH 7,3 para disección y extracción de sus órganos reproductores, con ayuda de una lupa, usando tijeras quirúrgicas, pinzas, alfileres entomológicos y base de parafina sólida en caja de petri.

El aparato reproductor de hembras y de machos del predador fue separado y colocado en tubos Eppendorf, que contenía solución fijadora de Zambonini (Stefanini et al. 1967). Las muestras fueron deshidratadas en serie alcohólica creciente e, incluidas, en histo-resina JB4. Con la ayuda del micrótomo automático fueron hechos cortes de $5 \mu \mathrm{m}$ de espesor, con navaja de vidrio. Las láminas con los cortes fueron coloreadas con hematoxilina y eosina y analizadas en microscopio de luz Olympus BX-50.

\section{RESULTADOS Y DISCUSIÓN}

\section{Sistema reproductor femenino}

La genitalia interna de las hembras está localizada en el abdomen, encima del intestino y está cubierta por el cuerpo graso; tiene coloración blanca y presenta un par de ovarios conectados a un par de oviductos laterales, que se unen formando el oviducto común (Figura 1A).

Cada ovario está compuesto por siete ovaríolos, unidos entre sí por filamentos, formando una estructura compacta. Cada ovaríolo, se asemeja a un filamento largo y consiste de: a) un germario apical, donde existen células indiferenciadas que se pueden dividir en ovocitos o trofocitos; b) un trofario, donde se encuentran los trofocitos o células nutridoras, los cuales, se unen a los ovocitos por cordones nutritivos y, c) un vitelario proximal, donde los ovocitos reciben vitelo. Cada ovaríolo está recubierto por la membrana peritoneal (Holtz, 2006) (Figuras 1B y 1C).

En el vitelario existen ovocitos en diferentes estados de desarrollo, recubiertos por una camada única de células foliculares, que presentan forma de columna con dos núcleos y nucléolo bien desarrollado; la función de ellas es secretar el corion del huevo (Figuras 1D y 1E).

La estructura del sistema reproductor de la hembra de S. cincticeps concuerda con la estructura típica de los representantes del suborden Heteroptera (Chapman, 1998). Los ovarios son del tipo meroístico-telotrófico (Buning, 1994; Chapman, 1998; Triplehorn \& Johnson, 2004), porque presentan trofocitos o células nutridoras y porque estos permanecen en el germario y se ligan a los ovocitos, por cordones nutritivos citoplasmáticos. En esta especie, el número de ovaríolos son siete, que están de acuerdo con la morfología reproductiva para Heteroptera, que va de cuatro a siete (Buning, 1994; Simiczyjew et al. 1998; Adams, 2000; Triplehorn \& Johnson, 2004) y que varían en relación con el tamaño y el estilo de vida del artrópodo, así como de la posición taxonómica del insecto estudiado (Chapman, 1998).

Lemos et al. (2005) señalan que en la especie $P$. nigrispinus, los ovaríolos contienen una serie linear de ovocitos en estados sucesivos de desarrollo, con los más avanzados en la posición proximal y más distantes del germario, la misma situación se observó en S. cincticeps, donde los ovocitos desarrollados se encontraron en la región del vitelario.

Las dos últimas fases de maduración de los ovocitos exigen una actividad especial de las células foliculares, que son responsables por controlar el acceso de proteínas y de nutrientes para el interior de los ovocitos y por secretar el corion de los mismos (Lemos et al. 2003). Las células foliculares halladas alrededor de los ovocitos de S. cincticeps, presentan dos núcleos y un nucléolo bien desarrollado, lo que demuestra que ellas tienen bastante actividad para contribuir en la nutrición y en el desarrollo total del ovocito.

Según Lundgren (2011), el conocimiento de la ecología reproductiva de estos insectos depredadores está intrínsecamente ligado a la fisiología y a la morfología de la hembra y sus huevos. Los parámetros de madurez y de diapausa reproductiva y fecundidad de las hembras de los depredadores de Heteroptera tienen gran influencia sobre el éxito de la tasa de crecimiento demográfico y reproductivo; por lo tanto, el conocer el estatus fisiológico reproductivo de las hembras de los depredadores pertenecientes a Heteroptera, el cual depende de varios factores fisiológicos, como el tipo de apareamiento, edad y tamaño, y el estado nutricional del depredador, puede proporcionar las bases para su empleo en un programa de control biológico de varias plagas agrícolas y forestales.

El tipo de alimentación suministrado a los chinches predadores de Pentatomidae pueden influenciar en el desarrollo de los ovarios y, consecuentemente, en su éxito reproductivo, como fue determinado para los depredadores Perillus bioculatus (F.) y Brontocoris tabidus (Signoret) (Heteroptera: Pentatomidae) (Adams, 2000; Lemos et al. 2009).

\section{Sistema reproductor masculino}

La genitalia interna de los machos, se localiza en el abdomen, encima del intestino y está cubierta por el cuerpo graso; presenta un par de testículos de color rojo y forma redonda, dos canales deferentes de color rojo y forma de filamentos largos y un ducto eyaculatorio de color amarillo (Figura 2A).

Los testículos están formados por cinco folículos, cuatro de ellos están bien desarrollados y uno es de menor tamaño, todos cubiertos por una membrana peritoneal, haciendo que los testículos estén fuertemente compactados (Figura 2B). Cada folículo está dividido en cuatro regiones: el germario, la zona de crecimiento, la zona de maduración y reducción y la zona de trasformación (Chapman, 1998). El germario 

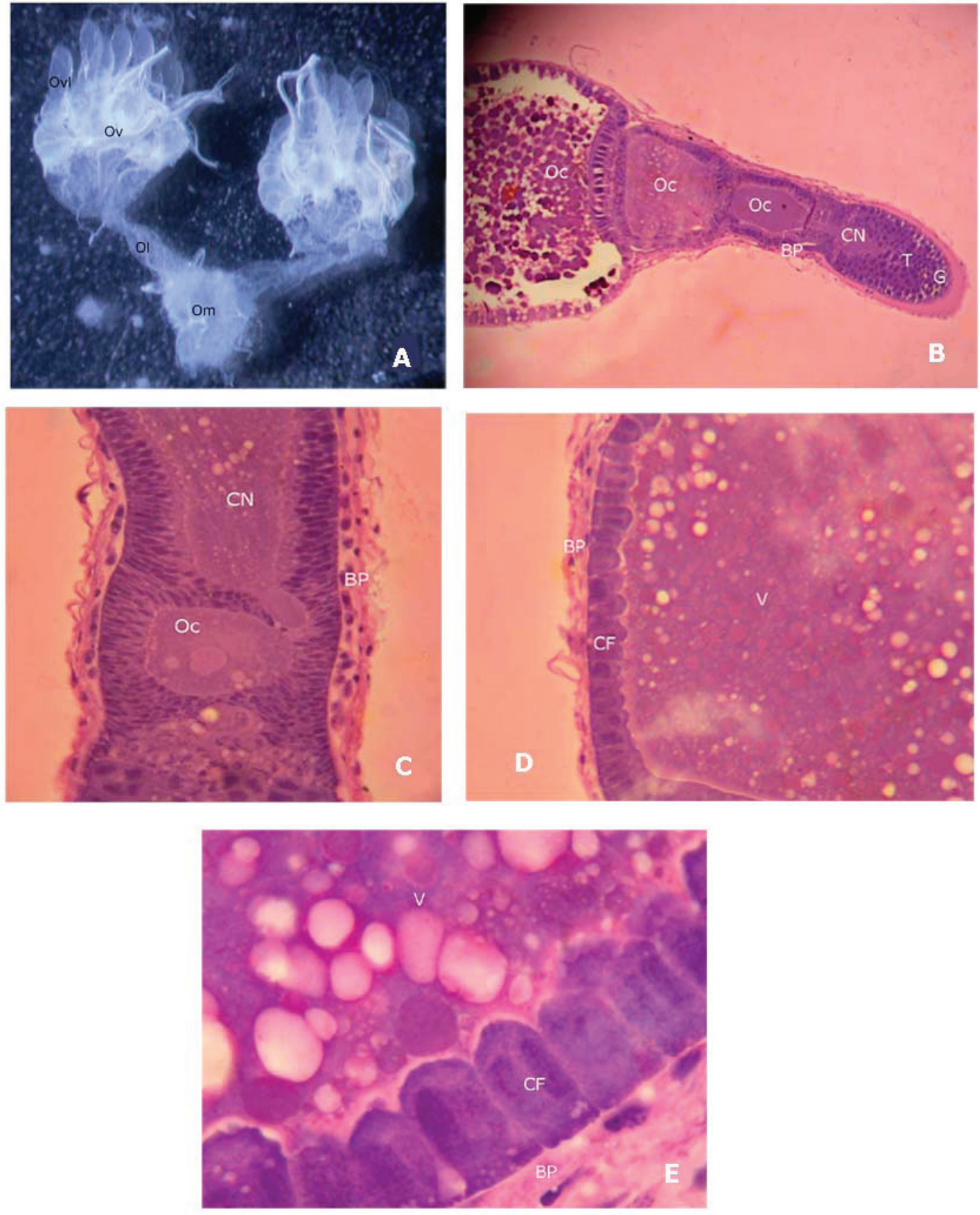

Figura 1. Vista general del sistema reproductor femenino de Supputius cincticeps (Heteroptera: Pentatomidae), presentando: (A) $\mathrm{Ov}=$ Ovarios, Ovl= Ovariolo, $\mathrm{Ol}=$ Oviducto lateral y $\mathrm{Om}=$ Oviducto común. (B) y (C) Corte longitudinal de folículo. $\mathrm{G}=$ Germario; $\mathrm{T}=$ Trofario; $\mathrm{CN}=$ Cordón nutritivo; $\mathrm{BP}=$ Membrana peritoneal; Oc= Ovocitos en diferentes estados de desarrollo. (D) y (E) Corte longitudinal del ovocito en desarrollo, presentando $\mathrm{BP}=$ Membrana peritoneal; $\mathrm{CF}=$ Células foliculares (columnares) y V= Gránulos de vitelo. 

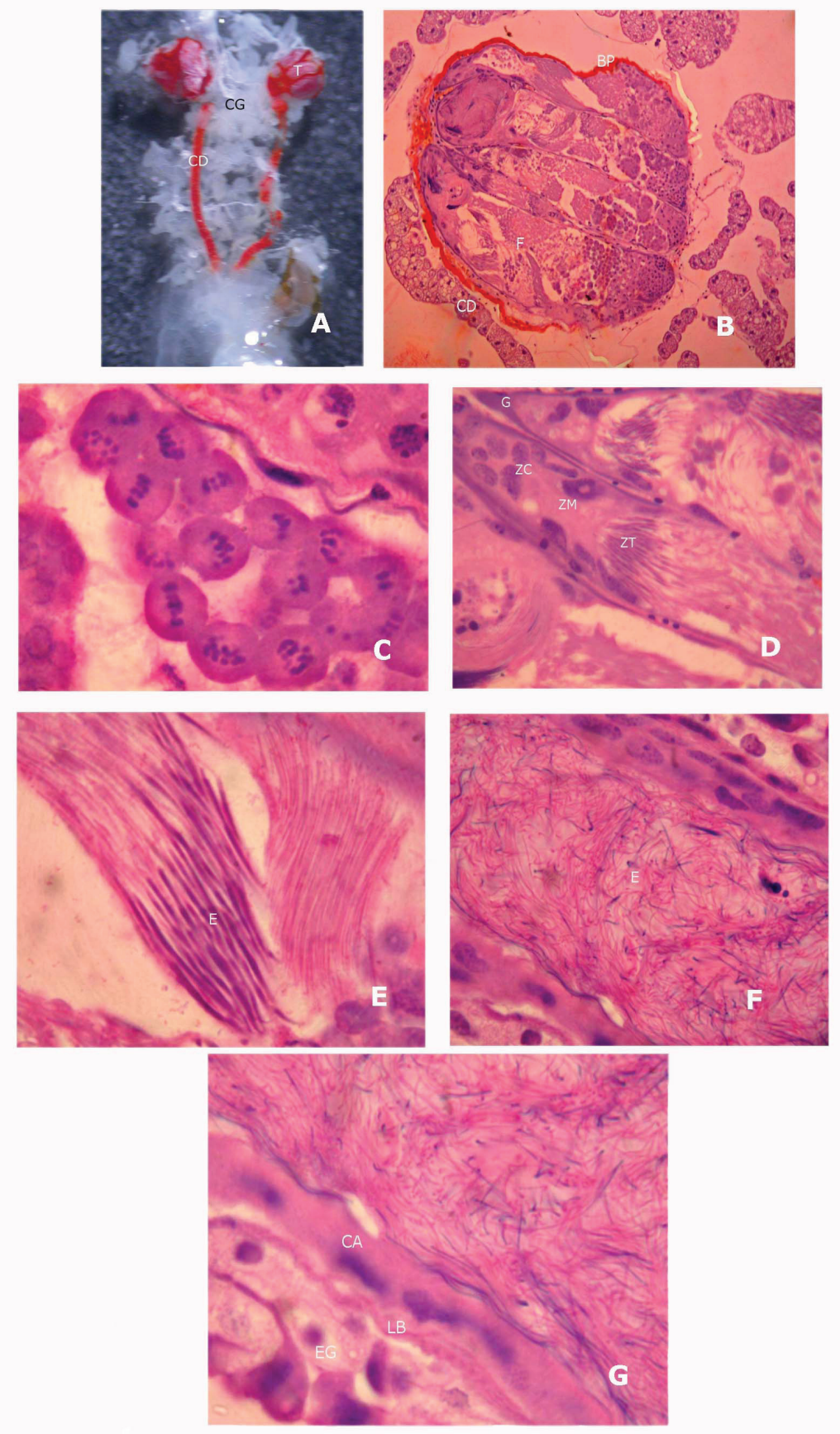

Figura 2. Vista general de la genitalia interna del macho de la especie Supputius cincticeps (Heteroptera: Pentatomidae), presentando: (A) $\mathrm{T}=$ Testículos, $\mathrm{CG}=$ Cuerpo graso, $\mathrm{CD}=$ Canal deferente. (B) $\mathrm{F}=$ Folículos; $\mathrm{CD}=$ Canal deferente; $\mathrm{BP}=$ Membrana peritoneal. (C) Zona de maduración dentro del folículo, donde ocurre el proceso de meiosis. (D) Estructura de los folículos: $\mathrm{G}=$ Germario; $\mathrm{ZC}=$ Zona de crecimiento; $\mathrm{ZM}=$ Zona de maduración; $\mathrm{ZT}=$ Zona de transformación. (E) $\mathrm{E}=$ Espermatozoides en masa dentro del folículo. (F) $\mathrm{E}=$ espermatozoides dentro del canal deferente. (G) Corte longitudinal del canal deferente, mostrando $\mathrm{EG}=$ Epitelio grueso; $\mathrm{LB}=$ Lámina basal y $\mathrm{CA}=$ Células con núcleos achatados. 
está conformado por células germinales que producen los espermatogonios, que son células que se dividen mitóticamente para producir los espermatocitos, las cuales, a su vez, producen espermátidas (Chapman, 1998); en la zona de transformación, las espermátidas se transforman en espermatozoides (Figura 2C).

Dentro de la zona de maduración del folículo, se encuentran espermatozoides filamentosos, largos y agrupados (Figura 2D); cuando los espermatozoides son liberados en el canal deferente, se encuentran totalmente desorganizados y en gran cantidad (Figura 2E). El canal deferente tiene forma de un tubo largo, formado por tres láminas (de dentro para fuera), una de células con núcleo achatado, una lamina basal y un epitelio grueso (Figura 2F).

La estructura de la genitalia interna del macho de S. cincticeps es similar a aquella encontrada en la especie $P$. nigrispinus, que se caracteriza por tener coloración rojo intenso y forma redondeada para los testículos y la misma coloración y forma de filamentos largos, para los canales deferentes (Lemos et al. 2005).

Cada testículo de la especie $P$. nigrispinus está compuesto de cuatro a seis folículos; cuando presenta seis folículos, cuatro de ellos son más largos y dos más atrofiados (Lemos et al. 2005); en S. cincticeps, se halló un patrón similar de cinco folículos, cuatro de ellos bien desarrollados y uno más corto. Para el caso de Nezara viridula (L.) (Heteroptera: Pentatomidae), cada testículo tiene seis folículos aplanados (Pendergrast, 1956).

La estructura de los folículos de la especie en estudio permitió observar espermatocitos en diferentes estados de maduración, demostrando la constante actividad en la producción de espermatozoides por los testículos, el mismo patrón fue verificado en $P$. nigrispinus (Lemos et al. 2005).

Los espermatozoides de $S$. cincticeps son filamentosos y largos, concordando con la forma de los espermatozoides de la mayoría de los insectos (Chapman, 1998; Triplehorn \& Johnson, 2005). De acuerdo con Chapman (1998), los espermatozoides son inactivos en los canales deferentes y son cargados por movimientos peristálticos hasta la vesícula seminal, donde son almacenados, esto explica la gran cantidad y la desorganización de espermatozoides encontrados en el canal deferente de $S$. cincticeps.

Agradecimientos: A la Fundação de Amparo á Pesquisa do Estado de Minas Gerais (FAPEMIG), Brazil, por el financiamiento de la investigación. Conflictos de intereses: El artículo fue preparado y revisado con la participación de todos los autores, quienes declaramos que no existe ningún conflicto de intereses que ponga en riesgo la validez de los resultados presentados

\section{BIBLIOGRAFÍA}

1. ADAMS, T.S. 2000. Effect of diet and mating status on ovarian development in a predaceous stink bug Perillus bioculatus (Hemiptera: Pentatomidae). Ann. Entomol. Soc. Am. 93:529-535.

2. BUNING, J. 1994. The insect ovary-Ultrastructure, previtellogenic growth and evolution. London: Chapman \& Hall. p.311-324.

3. CHAPMAN, R.F. 1998. The insects: Structure and Function. Cambridge University, Cambridge, 770p.

4. DE CLERCQ, P.; WYCKHUYS, K.; OLL-VEIRA, H.N.; KLAPWIJK, J. 2002. Predation by Podisus maculiventris on different life stages of Nezara viridula. Florida Entomologist. 85(1):197-202.

5. HAPP, G.M. 1992. Maturation of de male reproductive system and is endocrine regulation. Ann. Rev. Entomol. 37(1):303-320.

6. HOLTZ, A.M.; ZANUNCIO, J.C.; MARINHO, J.S.; PRATISSOLI, D.; PALLINI, A.; PEREIRA, D.J. 2006. Características biológicas de adultos de Podisus nigrispinus e Supputius cincticeps (Hemiptera: Pentatomidae) alimentados com Thyrinteina arnobia (Lepidoptera: Geometridae). Idesia. 24(2):41-48.

7. JERVIS, M.A.; COPLAND, M.J.W.; HARVEY, J.A. 2005. The life-cycle. En: JERVIS M.A. ed. Insects as natural enemies: A practical perspective. Springer (Dordrecht). p.72-165.

8. KSIAZKIEWICZ-KAPRALSKA, M. 1985. Organization of the trophic chamber of homopteran insects. 1. Cercopidae: Cicadomorpha. Cytobios 42(167168):133-145.

9. KSIAZKIEWICZ-KAPRALSKA, M. 1991. Organization of the trophic chamber of homopteran insects. 2. Membracidae: Cicadomorpha. Cytobios. 66(265):113-119.

10. LEMOS, W.P.; RAMALHO, F.S.; SERRAO, J.E.; ZANUNCIO, J.C. 2003. Effects of diet on development of Podisus nigrispinus (Dallas) (Heteroptera: Pentatomidae), a predator of the cotton leafworm. J. Appl. Entomol. 127(7):389-395. 
11. LEMOS, W.P.; RAMALHO, F.S.; SERRAO, J.E.; ZANUNCIO, J.C. 2005. Morphology of female reproductive tract of the predator Podisus nigrispinus (Dallas) (Heteroptera: Pentatomidae) fed on different diets. Braz. Arch. Biol. Techn. 48(1):129-138.

12. LEMOS, W.P.; ZANUNCIO J.C.; RAMALHO, F.S.; SERRÃO, J.E. 2009. Fat body of the zoophytophagous predator Brontocoris tabidus (Het.: Pentatomidae) females: Impact of the herbivory and age. Micron 40(5-6):635-638.

13. LUNDGREN,J.G.2011. Reproductive ecology of predaceous Heteroptera. Biological Control. 59(1):37-52.

14. NIJHOUT, H.F. 1994. Insect hormone. Princeton University Press, Princeton. 280p.

15. OLIVEIRA, J.E.M.; TORRES, J.B.; CARRANO-MOREIRA, A.F.; BARROS, R. 2002. Effects of cotton and tomato plants as complementary food on development and reproduction of the predator Podisus nigrispinus (Dallas) (Heteroptera: Pentatomidae). Neotrop. Entomol. 31(1):101-108.

16. PENDERGRAST, J.G. 1956. The male reproductive organs of Nezara viridula (L.) with a preliminary account of their development (Heteroptera: Pentatomidae). Trans. R. Soc. N.Z. 84:139-146.

17. PEREIRA, A.I.A.; RAMALHO, F.DES.; MALAQUÍA, J.B.; BANDEIRA, C.M.; SILVA, J.P.S.; ZANUNCIO, J.C. 2008. Density of Alabama arguillacea larvae affects food extraction by females of Podisus nigrispinus. Phytoparasitica. 6:84-94.

18. SANTOS, G.P.; ZANUNCIO, J.C.; SANTANA D.Q.L.; ZANUNCIO, T.V. 1993. Descrição das pragas desfolhadoras. p.12-66. In: Zanuncio, J.C. (Ed.). Manual de pragas em florestas- Lepidoptera desfolhadoras de eucalypto: biologia, ecologia e controle. Vol. 1. Folha de Viçosa, Viçosa, 140p.

19. SIMICZYJEW, B.; OGORZALEK, A.; STYS, A. 1998. Heteroptera ovaries: variations on the theme. Folia Histochem. Cytobiol. 36:147-156.

20. STEFANINI, M.; DE MARTINO, C.; ZAMBONI, L. 1967. Fixation of ejaculated spermatozoa for electron microscopy. Nature. 216(211):173-174.

21. SZKLARZEWICZ, T. 1998. The ovaries of scale insects (Hemiptera: Coccidae). Morphology and phylogenetic conclusions. Folia. Histochem. Cyto. 36(4):157-165.

22. TRIPLEHORN, C.A.; JOHNSON, N.F. 2005. Borror and DeLong's Introduction to the Study of Insects. Thomson Brooks/Cole (USA). 888p.

23. ZANUNCIO, J.C.; BRAGANÇA, M.A.L.; DIAZ, J.L.S.; SARTÓRIO, R.C. 1992. Avaliação dos parâmetros de fecundidade de Podisus connexivus (Heteroptera: Pentatomidae) de diferentes pesos. Rev. Ceres. 39:591-596.

24. ZANUNCIO, J.C.; SAAVEDRA, J.L.D.; ZANUNCIO, T.V.; SANTOS, G.P. 1996. Incremento en el peso de ninfas y adultos de Podisus nigrispinus (Heteroptera: Pentatomidae) alimentados con dos tipos de larva. Rev. Biol. Trop. 44(1):241-245.

25. ZANUNCIO, J.C.; ZANUNCIO, T.V.; GUEDES, R.N.C.; RAMALHO, F.S. 2000. Effect of feeding on three Eucalyptus species on the development of Brontocoris tabidus (Heteroptera: Pentatomidae) fed with Tenebrio molitor (Coleoptera: Tenebrionidae). Biocontrol. Sci. Techn. 10(4):443450.

26. ZANUNCIO, J.C.; MOLINA-RUGAMA, A.J.; SANTOS, G.P.; RAMALHO, F.S. 2002. Effect of body weight on fecundity and longevity of the stinkbug predator Podisus rostralis. Pesqui. Agropecu. Bras. 37(9):1225-1230.

27. ZANUNCIO, T.V.; ZANUNCIO, J.C.; SERRAO, J.E.; MEDEIROS, R.S.; PINON, T.B.; SEDIYAMA, C.A. 2005a. Fertility and life expectancy of the predator Supputius cincticeps (Heteroptera: Pentatomidae) exposed to sublethal doses of permethrin. Biol. Res. 38(1):31-39.

28. ZANUINCIO, J.C.; BESERRA, E.B.; MOLINA-RUGAMA, A.J.; ZANUNCIO, T.V.; PINON, T.B.M.; MAFFIA, M.P. 2005b. Reproduction and longevity of Supputius cincticeps (Heteroptera: Pentatomidae) fed with larvae of Zophobas confusa, Tenebrio molitor (Coleoptera: Tenebrionidae) or Musca domestica (Diptera: Muscidae). Braz. Arch. Biol. Techn. 48:771-777.

Recibido: Septiembre 18 de 2011

Aceptado: Marzo 3 de 2012 